\title{
Ultrasound guidance to perform intra-articular injection of gadolinium-based contrast material for magnetic resonance arthrography as an alternative to fluoroscopy: the time is now
}

\author{
Carmelo Messina ${ }^{1}$ - Giuseppe Banfi ${ }^{2,3}$ - Alberto Aliprandi ${ }^{4} \cdot$ Giovanni Mauri $^{4,6}$. \\ Francesco Secchi ${ }^{4,5}$ - Francesco Sardanelli ${ }^{4,5}$ - Luca Maria Sconfienza ${ }^{4,5}$
}

Received: 27 April 2015 / Revised: 9 July 2015 / Accepted: 23 July 2015 /Published online: 8 August 2015

(C) European Society of Radiology 2015

\begin{abstract}
Magnetic resonance (MR) imaging has been definitively established as the reference standard in the evaluation of joints in the body. Similarly, magnetic resonance arthrography has emerged as a technique that has been proven to increase significantly the diagnostic performance if compared with conventional MR imaging, especially when dealing with fibrocartilage and articular cartilage abnormalities. Diluted gadolinium can be injected in the joint space using different approaches: under palpation using anatomic landmarks or using an imaging guidance, such as fluoroscopy, computed tomography, or ultrasound. Fluoroscopy has been traditionally used, but the involvement of ionizing radiation should represent a remarkable limitation of this modality. Conversely, ultrasound has emerged as a feasible, cheap, quick, and radiation-free modality that can be used to inject joints, with comparable accuracy of fluoroscopy. In the present paper, we discuss the advantages and disadvantages of using fluoroscopy or ultrasound in injecting gadolinium-based contrast agents in joints to perform magnetic
\end{abstract}

Luca Maria Sconfienza

io@lucasconfienza.it

Scuola di Specializzazione in Radiodiagnostica, Università degli Studi di Milano, Via Mangiagalli 31, 20100 Milano, Italy

2 IRCCS Istituto Ortopedico Galeazzi, Via R. Galeazzi 4, 20161 Milano, Italy

3 Università Vita-Salute San Raffaele, Via Olgettina 58, 20132 Milano, Italy

4 Dipartimento di Scienze Biomediche per la Salute, Università degli Studi di Milano, Via Mangiagalli 31, 20100 Milano, Italy

5 Servizio di Radiologia, IRCCS Policlinico San Donato, Piazza Malan 1, 20097 San Donato, Milanese, Italy

6 Unità di Radiologia Interventistica, Istituto Europeo di Oncologia, Via Giuseppe Ripamonti 435, 20141 Milano, Italy resonance arthrography, also in view of the new EuroSAFE Imaging initiative promoted by the European Society of Radiology and the recent updates to the European Atomic Energy Community 2013/59 directive on the medical use of ionizing radiation.

Key Points

- Intra-articular contrast agent injection can be performed using different imaging modalities

- Fluoroscopy is widely used, but uses ionizing radiation

- Ultrasound is an accurate, quick, and radiation-free modality for joint injection

-X-rays should be avoided when other radiation-free modalities can be used

Keywords Magnetic resonance arthrography · Ultrasound · Fluoroscopy $\cdot$ Ionizing radiation $\cdot$ Radiation protection

\begin{tabular}{|c|c|}
\hline \multicolumn{2}{|c|}{ Abbreviations } \\
\hline MR & Magnetic resonance \\
\hline MRA & Magnetic resonance arthrography \\
\hline GBCM & Gadolinium-based contrast material \\
\hline US & Ultrasound \\
\hline UNSCEAR & $\begin{array}{l}\text { United Nations Scientific Committee on the } \\
\text { Effects of Atomic Radiation }\end{array}$ \\
\hline $\mathrm{CT}$ & Computed tomography \\
\hline EURATOM & European Atomic Energy Community \\
\hline
\end{tabular}

Magnetic resonance (MR) imaging has been definitively established as the reference standard in the evaluation of joints around the body, being capable of assessing thoroughly all articular components, such as tendons, ligaments, fibrocartilage, hyaline cartilage, and subchondral bone [1]. Similarly, MR arthrography (MRA) has emerged as a 
technique that has been proven to increase significantly the diagnostic performance if compared with conventional MR imaging, especially when dealing with fibrocartilage and articular cartilage abnormalities $[2,3]$. Even with the advent of higher field equipment, the diagnostic accuracy of MRA proved to be still superior compared to conventional MR imaging, as shown by Magee [4]. MRA is commonly used for imaging of the shoulder, but it is also used for imaging of other joints, including the hip, the wrist, the elbow, the ankle, and the knee [5-7].

MRA can be performed with a direct or indirect approach. While the indirect approach implies the intravenous administration of $0.2 \mathrm{mmol} / \mathrm{kg}$ of a gadolinium-based contrast material (GBCM) [2], direct MRA is obtained with the injection of a solution of diluted GBCM into the joint space. This allows for safely distending joint space, thus highlighting even minimal abnormalities that may be missed at conventional MRI $[2,3,8]$.

When ruling out certain diagnostic problems, direct MRA especially achieves almost the same sensitivity and specificity as the surgical reference standard [8-10]. For these reasons, in recent years, orthopaedic surgeons are increasingly prescribing this examination [11]. There are no official data regarding the number of MRA performed per year worldwide. In 2013 at our institution, a medium-sized university general hospital in Milan, Italy, out of 4,239 musculoskeletal MR examinations (excluding spine MRI), we performed 394 MRAs $(9.3 \%)$. This number is certainly expected to be higher in orthopaedic hospitals.

Diluted GBCM can be injected into the joint space using different approaches: under palpation using anatomic landmarks or using an imaging guidance, such as fluoroscopy, computed tomography (CT), MR imaging, or ultrasound (US) $[2,12]$. In particular, fluoroscopy is still used in most institutions, being a low-cost, efficient, and easy technique $[13,14]$. Nevertheless, fluoroscopic guidance has relevant disadvantages. First, it exposes both patients and operators to ionizing radiation. According to a report of the United Nations
Scientific Committee on the Effects of Atomic Radiation (UNSCEAR) issued in 2010, which offers a wide assessment of the magnitude of medical radiation exposure around the globe between 1997 and 2007, the average effective dose of an X-ray arthrogram is $0.17 \mathrm{mSv}$ [15]. This value is almost ten times higher than the effective dose of a postero-anterior chest $\mathrm{X}$-ray, whose average effective dose is $0.02 \mathrm{mSv}$ [16]. On the other hand, original data from Binkert et al. [17] showed an effective dose of $0.0015 \mathrm{mSv}$ for fluoroscopy-guided injection of the glenohumeral joint, a dose that may even decrease using full-digital X-ray image detector. At any rate, even though it may seem a very small radiation dose compared to other diagnostic and interventional procedures using ionizing radiations, the cumulative dose on general population will result in an unjustified radiation exposure. This is of even greater importance, as MRA is usually performed on young adult patients. Moreover, it should be noted that the shoulder and hip are the most commonly imaged joints, and they are very close to highly radiosensitive organs, namely the thyroid and gonads, respectively, which are, therefore, inevitably irradiated. In the literature, we were not able to find any traces of the use of thyroid and pelvic lead shields used in patients being injected to perform MRA contrast injection under fluoroscopic guidance, thus confirming the lack of awareness on this topic. Second, additional time for planning and performing the procedure may be needed, particularly if the fluoroscopic suite is distant from the MR room. Of note, for MRA, the degree of visualization of intra-articular structures depends on the time elapsed between contrast injection and MRI. MRA of the shoulder and hip should be performed within $90 \mathrm{~min}$, and MR arthrography of the wrist should be performed within 45 min after intraarticular injection to minimise absorption of the contrast agent and the loss of capsular distension [18].

Fluoroscopic guidance has some other drawbacks. During fluoroscopy, intra-articular needle placement should be confirmed with an injection of a small amount of iodinated contrast agents or adding iodinated contrast to the diluted

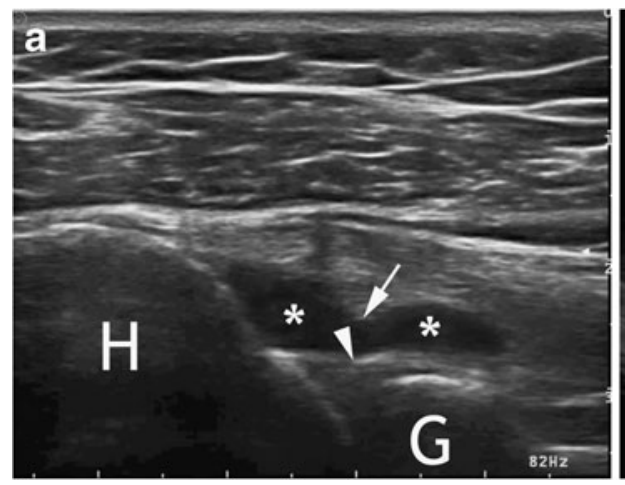

Fig. 1 Examples of ultrasound-guided intra-articular injections. (a) Posterior gleno-humeral injection with out-of-plane approach. Only the needle tip is visible (arrow). The posterior joint recess is distended by gadolinium-based solution (asterisks). $H=$ humerus, $G=$ glenoid,

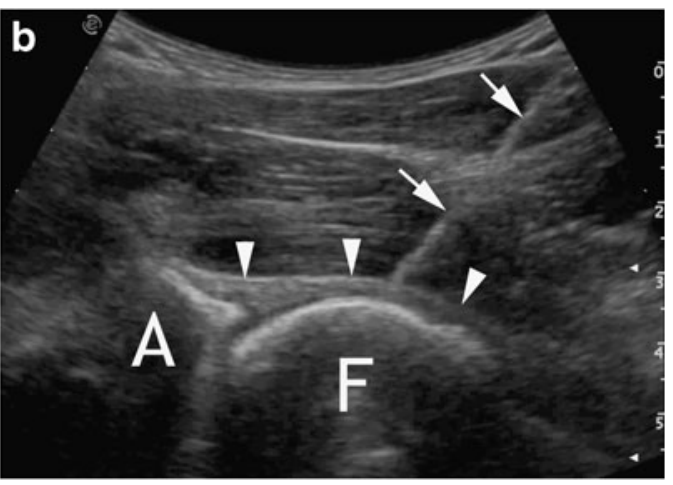

arrowhead $=$ glenoid labrum. $(\boldsymbol{b})$ Hip injection with in-plane approach. The needle shaft (arrows) is totally visible. $F=$ femoral head, $A=$ acetabulum, arrowheads $=$ joint capsule 
gadolinium. Nonetheless, iodinated contrast has been shown to influence the $\mathrm{T} 1$ and $\mathrm{T} 2$ relaxation times in a dosedependent manner, producing altered contrast-to-noise ratios [19-21]. Finally, although feasible and easy to perform, fluoroscopy still rests on two-dimensional images.

Conversely, US guidance of musculoskeletal interventional procedures has been extensively reported to be safe and feasible [22-24], and it is also known to have multiple advantages. First, no ionizing radiation is delivered, thus preserving the integrity of both patients and operators. Second, the needle tip can be seen more effectively with US compared to fluoroscopy, as the whole length of the needle can be followed on its major axis. Also, the needle tip can be clearly seen entering the joint space, thus implying that no iodinated contrast is needed to confirm correct needle positioning. Third, small portable US systems can be located directly in a medication room adjacent to the MR suite. This implies that a dedicated room is not needed for this procedure and the time between injection and MRA examination is reduced to minimum (Fig. 1).

Arthrography is generally considered a safe procedure with few serious complications [25]. Of these, septic arthritis is the most feared by physicians, although its incidence is reported to be as low as $0.003 \%$ [26]. Of note, a recent paper of Vollman et al. reports three cases of MRA-related infections followed by fluoroscopic injection of gadolinium. Authors propose that contamination source was related to barium swallow studies which preceded the arthrogram injections in the same room [27]. However, since the injection procedure itself is very similar when using both fluoroscopic or US guidance, complication rate is expected to be comparable. However, no papers are available comparing directly these two injection modalities.

Several papers evaluated the feasibility and accuracy of US-guided GBCM injection for MRA in different districts, also comparing US to other arthrographic techniques such fluoroscopy and CT [14, 28, 29]. They conclude that US is an effective alternate guidance modality for the injection of gadolinium into shoulder, hip, knee, and wrist joints for MRA. For the glenohumeral joint, $\mathrm{Ng}$ et al. concludes that US injection is comparable with fluoroscopic-guided injection in terms of patient comfort, time, and efficiency [14]. The success of shoulder injection seems to be comparable both using anterior and posterior approaches [30]. Similar results are shown for US guidance when used for MRA of the hip [29]. A recent review by Berkoff et al. investigated the effect of US guidance on different variables (needle placement, cost-effectiveness, clinical outcomes) in comparison with anatomical landmarkguided intra-articular injections [31]. Comparing 13 studies, they reported that US guidance of knee injections resulted in better needle placement accuracy compared to anatomical guidance (95.8 \% versus $77.8 \%$, respectively). Also, the authors refer to a randomized controlled trial by Sibbit et al. in which they compared the clinical outcome of US-guided versus anatomical guided arthrocentesis and intra-articular corticosteroid injection, concluding that US guidance determined $48 \%$ less procedural pain and $46 \%$ less pain at 2 weeks [32]. US represents a feasible technique also for wrist and ankle intra-articular injections with a $100 \%$ success rate, as shown by Choudur et al. in 2011 [33]. US-guided intra-articular elbow joint injections resulted in better intra-articular delivery than palpation-guided, as confirmed by Kim et al [34]. Clearly, the main advantage is that ionizing radiation is not involved.

One may argue that the use of fluoroscopy to inject patients is an approach of the past. To clarify this aspect, we empirically performed a quick search on the PubMed using "MR arthrography" as a keyword, including papers published in 2013. Out of 130 retrieved records, we selected 29 papers in which the guidance technique is clearly illustrated. Excluding one paper in which injection was performed using palpation guidance, the use of US is reported in six papers only $(21 \%)$, while the majority of authors (22 papers, $76 \%$ ) still prefer to use fluoroscopy to inject patients (unpublished data). Nevertheless, we included in this search all the anatomic districts. In certain regions, such as the wrist, the use of fluoroscopy may have a supplementary diagnostic value, even though it can be considered a different and separate examination from injection guidance itself. In other words, if fluoroscopy (i.e., conventional arthrography) is needed for whatever diagnostic purpose, it should be performed (and possibly reimbursed) as a separate examination, but the use of fluoroscopy with the mere purpose of joint injection is questionable. One reason for such a widespread use of fluoroscopic guidance may be because the use of US in the musculoskeletal system has only recently increased, especially in countries such as the United States, so that the preferred method to inject is traditionally fluoroscopy [35]. For the same reason, it is possible that radiologists feel more confident with fluoroscopy compared to US.

In the European Union, the exposure of patients to ionizing radiation for medical purposes has been traditionally ruled by the European Atomic Energy Community (EURATOM) with its 97/43/EURATOM directive dated 1997, adopted in the following years by all European countries. This directive was recently reviewed and updated, and the new text is contained in the directive 2013/59/EURATOM, which should be adopted by European countries during the coming years [36]. This directive makes the European Union unique, because no other country has something similar. In the United States, each single state has different regulatory mechanisms for the medical use of X-rays [36]. In chapter VII of 2013/59/EURATOM, entitled "Justification to medical radiation exposure" it is clearly stated that exposure should "take into account the efficacy, benefits, and risks of available alternative techniques having the same objective but involving no or less exposure to ionizing radiation" [36]. In other words, if an imaging modality without ionizing radiation can be reasonably used to achieve the same 
result, it should be invariably used. To be realistic, there is no real reason why US should not replace fluoroscopy to inject joints, except the mere preference and inclination of the individual radiologist. To pursue this goal, the medical and scientific community should be more focused on the promotion of special educational courses to spread the practical use of US in performing these procedures.

To embrace this necessity for coordinating radiation safety actions in medical imaging, the European Society of Radiology launched the EuroSAFE Imaging campaign during the 2014 European Congress of Radiology, in order to support and strengthen medical radiation protection across Europe, interacting with the regulatory bodies of the European Union and trying to increase awareness of both patients and radiologist on radiation protection in clinical practice. Still, a lot of work has to be done [37].

In conclusion, US has emerged as an easy, cheap, effective, and radiation-free imaging modality to perform interventional procedures in the musculoskeletal system, particularly to perform joint injections for MRA. The use of fluoroscopy in this field should be restricted to very specific cases, namely when US is not available or cannot be used for certain reasons. Last, our community should promote education of musculoskeletal radiologists, both on the use of US in performing musculoskeletal procedures and on the potential damages and legal issues deriving from unjustified radiations exposure. "Progress is impossible without change, and those who cannot change their minds cannot change anything" (George Bernard Shaw).

Acknowledgments The scientific guarantor of this publication is Luca Maria Sconfienza. The authors of this manuscript declare no relationships with any companies, whose products or services may be related to the subject matter of the article. The authors state that this work has not received any funding. No complex statistical methods were necessary for this paper. Institutional review board approval was not required because the paper does not involve patients' data.

\section{References}

1. Sahin G, Demirtaș M (2006) An overview of MR arthrography with emphasis on the current technique and applicational hints and tips. Eur J Radiol 58:416-430

2. Waldt S, Bruegel M, Mueller D et al (2007) Rotator cuff tears: assessment with MR arthrography in 275 patients with arthroscopic correlation. Eur Radiol 17:491-498

3. Major NM, Browne J, Domzalski T, Cothran RL, Helms CA (2011) Evaluation of the glenoid labrum with 3-T MRI: is intraarticular contrast necessary? AJR Am J Roentgenol 196:1139-1144

4. Magee T (2009) 3-T MRI of the shoulder: is MR arthrography necessary? AJR Am J Roentgenol 192:86-92

5. Hodler J (2008) Technical errors in MR arthrography. Skelet Radiol 37:9-18

6. Sutter R, Zubler V, Hoffmann A et al (2011) Hip MRI: how useful is intraarticular contrast material for evaluating surgically proven lesions of the labrum and articular cartilage? AJR Am J Roentgenol 202:160-169
7. Lee RK, Ng AW, Tong CS et al (2013) Intrinsic ligament and triangular fibrocartilage complex tears of the wrist: comparison of MDCT arthrography, conventional 3-T MRI, and MR arthrography. Skelet Radiol 42:1277-1285

8. Elentuck D, Palmer WE (2004) Direct magnetic resonance arthrography. Eur Radiol 14:1956-1967

9. Ferrari FS, Governi S, Burresi F, Vigni F, Stefani P (2002) Supraspinatus tendon tears: comparison of US and MR arthrography with surgical correlation. Eur Radiol 12:1211-1217

10. Chundru U, Riley GM, Steinbach LS (2009) Magnetic resonance arthrography. Radiol Clin N Am 47:471-494

11. Kassarjian A (2012) Current concepts in MR and CT arthrography. Semin Musculoskelet Radiol 16:1-2

12. Graves MJ, Wakely S, Bearcroft PW et al (2008) MR-guided direct arthrography of the hip. J Magn Reson Imaging 28:462-465

13. Steinbach LS, Palmer WE, Schweitzer ME (2002) Special focus session. MR arthrography. Radiographics 22:1223-1246

14. Ng AW, Hung EH, Griffith JF, Tong CS, Cho CC (2013) Comparison of ultrasound versus fluoroscopic guided rotator cuff interval approach for MR arthrography. Clin Imaging 37: $548-553$

15. United Nations Scientific Committee on the Effects of Atomic Radiation (2008) Sources and Effects of Ionizing Radiation. Report, Volume 1. Available at page 76 of http://www.unscear. org/docs/reports/2008/09-86753_Report_2008_Annex_A.pdf Accessed on 30 March 2015

16. Mettler FA Jr, Huda W, Yoshizumi TT, Mahesh M (2008) Effective doses in radiology and diagnostic nuclear medicine: a catalog. Radiology 248:254-263

17. Binkert CA, Verdun FR, Zanetti M, Pfirrmann CW, Hodler J (2003) CT arthrography of the glenohumeral joint: CT fluoroscopy versus conventional CT and fluoroscopy-comparison of image-guidance techniques. Radiology 229:153-158

18. Andreisek G, Duc SR, Froehlich JM, Hodler J, Weishaupt D (2007) MR arthrography of the shoulder, hip, and wrist: evaluation of contrast dynamics and image quality with increasing injection-toimaging time. AJR Am J Roentgenol 188:1081-1088

19. Montgomery DD, Morrison WB, Schweitzer ME, Weishaupt D, Dougherty L (2002) Effects of iodinated contrast and field strength on gadolinium enhancement: implications for direct MR arthrography. J Magn Reson Imaging 15:334-343

20. Ugas MA, Huynh BH, Fox MG, Patrie JT, Gaskin CM (2014) MR arthrography: impact of steroids, local anesthetics, and iodinated contrast material on gadolinium signal intensity in phantoms at 1.5 and 3.0 T. Radiology 272:475-483

21. Kopka L, Funke M, Fischer U, Keating D, Oestmann J, Grabbe E (1994) MR arthrography of the shoulder with gadopentetate dimeglumine: influence of concentration, iodinated contrast material, and time on signal intensity. AJR Am J Roentgenol 163: 621-623

22. Fabbro E, Ferrero G, Orlandi D et al (2012) Rotator cuff ultrasoundguided procedures: Technical and outcome improvements. Imaging Med 4:649-656

23. Sconfienza LM, Viganò S, Martini C et al (2013) Double-needle ultrasound-guided percutaneous treatment of rotator cuff calcific tendinitis: tips \& tricks. Skelet Radiol 42:19-24

24. Sconfienza LM, Bandirali M, Serafini G et al (2012) Rotator cuff calcific tendinitis: does warm saline solution improve the short-term outcome of double-needle US-guided treatment? Radiology 262: 560-566

25. Hugo PC, Newberg AH, Newman JS, Wetzner SM (1998) Complications of Arthrography. Semin Musculoskelet Radiol 2: 345-348

26. Newberg AH, Munn CS, Robbins AH (1985) Complications of arthrography. Radiology 155:605-606 
27. Vollman AT, Craig JG, Hulen R, Ahmed A, Zervos MJ, van Holsbeeck M (2013) Review of three magnetic resonance arthrography related infections. World J Radiol 5:41-44

28. Perdikakis E, Drakonaki E, Maris T, Karantanas A (2013) MR arthrography of the shoulder: tolerance evaluation of four different injection techniques. Skelet Radiol 42:99-105

29. Kantarci F, Ozbayrak M, Gulsen F, Gencturk M, Botanlioglu H, Mihmanli I (2013) Ultrasound-guided injection for MR arthrography of the hip: comparison of two different techniques. Skelet Radiol 42: $37-42$

30. Zwar RB, Read JW, Noakes JB (2004) Sonographically guided glenohumeral joint injection. AJR Am J Roentgenol 183:48-50

31. Berkoff DJ, Miller LE, Block JE (2012) Clinical utility of ultrasound guidance for intra-articular knee injections: a review. Clin Interv Aging 7:89-95

32. Sibbitt WL Jr, Peisajovich A, Michael AA et al (2009) Does sonographic needle guidance affect the clinical outcome of intraarticular injections? J Rheumatol 36:1892-1902
33. Choudur HN, Ellins ML (2011) Ultrasound-guided gadolinium joint injections for magnetic resonance arthrography. J Clin Ultrasound 39:6-11

34. Kim TK, Lee JH, Park KD, Lee SC, Ahn J, Park Y (2013) Ultrasound versus palpation guidance for intra-articular injections in patients with degenerative osteoarthritis of the elbow. J Clin Ultrasound 41:479-485

35. Sharpe RE, Nazarian LN, Parker L, Rao VM, Levin DC (2012) Dramatically increased musculoskeletal ultrasound utilization from 2000 to 2009 , especially by podiatrists in private offices. J Am Coll Radiol 9:141-146

36. European Commission. 2013/59/EURATOM directive. Available at http://eur-lex.europa.eu/legal-content/EN/TXT/? uri=uriserv:OJ.L_.2014.013.01.0001.01.ENG. Accessed on 30 March 2015

37. European Society of Radiology EuroSAFE Imaging initiative. Available at http://www.eurosafeimaging.org/about. Accessed on 30 March 2015 